\title{
A STUDY ON MAGNETIC RESONANCE IMAGING FINDINGS IN CHILDHOOD EPILEPSY
}

\author{
Rehna K. Rehman'1, Sheela Theparambil Alikunju²
}

1Resident, Department of Paediatric Nephrology, St. John's Medical College, Bangalore.

${ }^{2}$ Associate Professor, Department of Paediatrics, Government Medical College, Thrissur, Kerala.

\section{ABSTRACT}

\section{BACKGROUND}

Epilepsy is the most common brain disorder during childhood and it may be associated with serious conditions. Early recognition and treatment is essential for best possible outcome. MRI is the modality of choice for investigating epilepsy to detect the underlying aetiology. Different imaging techniques are available that can provide high levels of anatomical and metabolic data. MRI is an important tool in the assessment of children with inborn errors of metabolism.

The objective of this study is to study the prevalence of MRI findings and to evaluate the role of MRI in diagnosis and management of childhood epilepsy. The study also aims to compare MRI findings in children having normal neurological examination with those having the specified abnormalities. The clinical and EEG predictors of abnormal MRI were also identified.

\section{MATERIALS AND METHODS}

A total of 80 children between 2 - 14 years were admitted with history of seizure (other than symptomatic and febrile seizures) subjected to MRI and EEG were included. Data was obtained from observation and evaluation. Children were also grouped into two to compare MRI findings. The former with abnormal neurological examination and the latter having no neurological abnormalities. MRI and EEG findings were analysed using SPSS software version 18.

Settings- Cross-sectional study was conducted in the Department of Paediatrics, Government Medical College, Thrissur, from January - December 2013.

\section{RESULTS}

Mean age of the study population was estimated to be 7 years and that of the onset of seizures was 4.15 years. Abnormal MRI findings were found in 39 children (48.75\%). 35\% of children with normal examination and $62.5 \%$ with abnormal examination had abnormal MRI. The age of onset of seizures for children with abnormal MRI was significantly lower than that of those with nor mal MRI. The most common MRI abnormality detected was Malformations of Cortical Development. 74.3\% of children with history of Status Epilepticus had abnormal MRI findings, which were statistically significant. Abnormal EEG was found in $52.5 \%$ of the population with $10 \%$ showing focal abnormalities. Ten patients (12.5\%) were diagnosed with epileptic syndromes.

\section{CONCLUSION}

Magnetic Resonance Imaging abnormalities are common among epileptic children with abnormal neurological examination. Children with lower age of onset and with status epilepticus showed more abnormalities. MRI helps in the management of children with underlying surgically amenable structural lesions.

\section{KEYWORDS}

Paediatrics, Epilepsy, Seizures, MRI (Magnetic Resonance Imaging).

HOW TO CITE THIS ARTICLE: Rehman RK, Alikunju ST. A study on magnetic resonance imaging findings in childhood epilepsy. J. Evolution Med. Dent. Sci. 2018;7(01):116-121, DOI: 10.14260/jemds/2018/25

\section{BACKGROUND}

Epilepsy is a disorder of the brain characterised by an enduring predisposition to generate seizures and the neurobiological, cognitive, psychological and social consequences of this condition.(1) For epidemiologic purposes, epilepsy is defined as the occurrence of two or more unprovoked seizures occurring one after the other more than twenty-four hours apart. "Unprovoked" here implies that it need not be a direct consequence of any acute illness, fever or brain injuries. ${ }^{(2)}$

'Financial or Other Competing Interest': None.

Submission 16-11-2017, Peer Review 13-12-2017,

Acceptance 19-12-2017, Published 01-01-2018.

Corresponding Author:

Dr. Sheela Theparambil Alikunju,

Associate Professor,

Department of Paediatrics,

Government Medical College,

Thrissur, Kerala.

E-mail: sheelatheparambil@gmail.com

DOI: $10.14260 /$ jemds $/ 2018 / 25$
A "seizure" is defined as a transient appearance of signs or symptoms due to abnormal, excessive or synchronous neuronal activity in the brain. Most of the significant epilepsies have their onset in childhood.(3)

An Epileptic Syndrome is a disorder that manifests as one or more specific seizure types with a specific age of onset as well as a specific prognosis. The age of onset will aid in diagnosing certain epileptic syndromes specific to that age and identifying the aetiology.(4) In symptomatic epilepsy, the underlying brain disorder is the aetiology of seizures. A presumed underlying brain disorder causing the epilepsy and affecting the neurological disorder is termed as Cryptogenic epilepsy or Presumed symptomatic epilepsy.

The prevalence of epilepsy in studies from India shows a rate of 5.59 per 1000 population with no gender or geographical differences.(5) In Kerala, this is 4.7 cases per 1000 population.(6) The ILAE (International League Against Epilepsy) has taken efforts to modify the classification scheme timely depending on the most latest developments. ${ }^{(7)}$ The old classifications of idiopathic, symptomatic and cryptogenic were replaced by genetic, structural or metabolic 
and unknown. $(8,9)$ The latest classification of seizure type by ILAE 2017, basic version include focal onset with awareness and with impaired awareness, generalized onset and unknown onset types. Each type is further divided into motor and non-motor types. Focal to bilateral tonic-clonic and unclassified types forms the subclasses.(10)

Magnetic resonance imaging is the best modality for neuroimaging in paediatric epilepsy.(11,12) Routine neuroimaging in children should include T1 weighted imaging of the whole brain to detect midline developmental anomalies.(13) Incomplete myelination in infants results in poor gray-white matter contrast, making detection of cortical anomalies difficult and hence may warrant a repeat imaging.(14) $\mathrm{T} 1$ and $\mathrm{T} 2$ weighted images should be obtained and CSF suppression Fluid Attenuated Inversion Recovery (FLAIR) is useful in identifying areas of gliosis.

Electroencephalogram (EEG) taken during the evaluation of any child with epilepsy helps in localisation, diagnosing certain syndromes and in predicting seizure recurrence.(13) Special EEG's like video EEG activation procedures may be required to detect abnormal discharges.(12,14) In this study, EEG is being evaluated as a tool for predicting abnormal MRI. MRI being a costly investigation should be used judiciously in a resource deficient country. There are only a few studies published from South India describing the MRI findings in childhood epilepsy. Though EEG, seizure semiology and neurological examination helps in localising the epileptic focus, MRI remains a sensitive tool of identifying and localising the underlying structural abnormality. $(15,16)$ Imaging helps in establishing aetiology, providing prognostic information and aids the treatment in children, particularly those with newly diagnosed epilepsy.(17) Developmental malformations are the most common abnormalities detected in children and adolescents by imaging.(18,19) Malformations of Cortical Development (MCD) are a heterogeneous group of disorders characterised by an abnormal architecture of the cerebral cortex and the most common cause of partial seizures as well as intractable epilepsy in children.(17) Focal Cortical Dysplasia (FCD) is characterised by heterogeneous lesions exhibiting abnormal neuronal or glial cells within a localised region of cerebral cortex. Out of the MCDs, they may escape detection by a routine MRI and sometimes even with special techniques.(20,21) Disorders of neuronal migration are due to under-migration, over-migration or ectopic migration and the respective examples are Classic Lissencephaly, Cobblestone Lissencephaly and Heterotopias. (20)

\section{MATERIALS AND METHODS Study Setting}

Department of Paediatrics, Government Medical College, Thrissur, from 1st January 2013 to 31st December 2013 (1 year).

Type of Study: Cross-sectional.

Study Population: Children admitted in the paediatric ward or attending paediatric OPD with a diagnosis of epilepsy.

Inclusion Criteria: Children in the age group 2 to 14 years with 2 or more unprovoked seizures in a timeframe of more than 24 hours.

Exclusion Criteria: Children with febrile seizures, acute symptomatic seizures, suspected neurometabolic disorders and those who were not subjected to MRI as part of evaluation.

Children below 2 years were not included because of the inconvenience in performing MRI in them due to problems with sedation.

Sample size was calculated using the formula $4 p q / d^{2}$, where $\mathrm{p}=$ prevalence and $\mathrm{q}=100-\mathrm{p}$.

$\mathrm{d}=$ maximum allowable error taken as $15 \%$ with a power of $85 \%$ and a significance level of $5 \%$.

Based on the prevalence of abnormal imaging in a study by Chaurasia et al, p was found to be $70.4 \% .{ }^{(22)}$

$\mathrm{p}=70.4, \mathrm{q}=100-\mathrm{p}=29.6, \mathrm{~d}=10.56$.

The calculated sample size was 75 and in this study, we have included 80 children. Tools- General Examination, Neurological Examination, Findings of Electroencephalogram (EEG) and Magnetic Resonance Imaging (MRI).

Status Epilepticus was defined as a continuous seizure activity or recurrent seizures without regaining consciousness and lasting for more than 30 minutes. Drug resistant epilepsy or medically intractable epilepsy is defined as the failure of sustained seizure control with adequate trial of two tolerated and appropriately chosen AED schedules. In this study, all children taking more than two AEDs were considered as having intractable epilepsy.

Abnormal examination findings were defined as significant developmental delay, mental retardation, children with dysmorphism, microcephaly and neurocutaneous markers, focal neurological deficits of any grade, visual or hearing impairment and pyramidal signs. Abnormal EEG was defined as any EEG abnormality detected to be of significance by the reporting neurologist. Abnormal MRI was defined as structural abnormalities in MRI, which can be congenital or acquired, excluding those due to immediate events like demyelination, acute stroke or infection.

Details were collected from all children with epilepsy in the age group of 2 to 14 years, admitted in the ward using proforma. The old and new cases were included and were classified into two groups. Those with normal neurologic examination and those with abnormalities in the same. Basic characteristics of the population were collected. Detailed natal, postnatal, family history and past history were taken. Information on seizure semiology, frequency and antiepileptic drugs were also enquired. Findings were entered after conducting a detailed general and neurological examination. Microcephaly was assessed using WHO charts.

A 30-minute interictal electroencephalogram was taken, both asleep and awake. Electrodes were placed using $10-20$ systems and the results were interpreted by a neurologist. Records were reviewed in the old cases. MRI was taken using 1.5 tesla MRI machine. T1 weighted, T2 weighted and FLAIR images were taken including coronal and axial sections. It was interpreted by a radiologist who was not informed about the diagnosis. The images were reviewed by the same radiologist in the old cases. Data was analysed using SPSS software version 18 .

\section{RESULTS}

Mean age of the population was 7 years and the median was 8 years $(n=80)$. Mean and median age of the normal group was 8 years $(n=40)$. Mean age of abnormal group was 6 years and median was 4.75 years $(n=40)$. 
The mean age of seizure onset in the population was 4.15 years. In the normal group, it was 5.7 years and 2.5 years in the abnormal group. Males constituted $58.7 \%$ and females $41.3 \%$ of the group. $82.5 \%$ of patients had generalised seizures, $10 \%$ had focal seizures and $7.5 \%$ had both generalised as well as focal seizures. GTCS (Generalised tonicclonic) was the predominant type seen equally among both the groups. The abnormal group had greater incidences of myoclonic seizures, while complex partial seizures were prevalent in the normal group. Epileptic syndromes constituted the diagnosis in $12.5 \%$ of the patients, where $20 \%$ had family history of seizures and $8.8 \%$ had family history of febrile seizures. Abnormal EEG was found in 52.5\% of patients with $10 \%$ showing focal abnormalities. $50 \%$ in normal group and 55\% in abnormal group had abnormal EEG.

Abnormal MRI findings were found in 39 children $(48.75 \%) .35 \%$ of children with normal examination and $62.5 \%$ of children with abnormal neurological examination had abnormal MRI. 39 children with abnormal MRI had 48 abnormal findings. Abnormal MRI findings were more among children with abnormal neurological examination. This difference was statistically significant.

\begin{tabular}{|l|l|l|l|}
\hline & $\begin{array}{l}\text { Normal } \\
\text { MRI }\end{array}$ & $\begin{array}{l}\text { Abnormal } \\
\text { MRI }\end{array}$ & Total \\
\hline $\begin{array}{l}\text { Normal } \\
\text { neurological } \\
\text { examination }\end{array}$ & $26(63.4 \%)$ & $14(35.9 \%)$ & 40 \\
\hline $\begin{array}{l}\text { Abnormal } \\
\text { neurological } \\
\text { examination }\end{array}$ & $15(36.6 \%)$ & $25(64.1 \%)$ & 40 \\
\hline Total & 41 (100\%) & $\mathbf{3 9 ( 1 0 0 \% )}$ & $\mathbf{8 0}$ \\
\hline
\end{tabular}

*Association between abnormal MRI and abnormal neurological examination. †The chi-square test value was $6.054, \mathrm{df}=1$ and $\mathrm{p}$ value was 0.014 .

\begin{tabular}{|l|l|l|l|}
\hline & Number & $\begin{array}{l}\text { Mean Age of } \\
\text { Onset in } \\
\text { Years }\end{array}$ & $\begin{array}{l}\text { Standard } \\
\text { Deviation }\end{array}$ \\
\hline Normal MRI & 41 & 5.088 & 4.018 \\
\hline $\begin{array}{l}\text { Abnormal } \\
\text { MRI }\end{array}$ & 39 & 3.57 & 3.57 \\
\hline
\end{tabular}

\section{Table 2}

$*_{\mathrm{t}}$ value $=2.255$ and $\mathrm{p}=0.027 . \dagger$ Mann-Whitney test $\mathrm{p}$ value $<$ 0.05 .

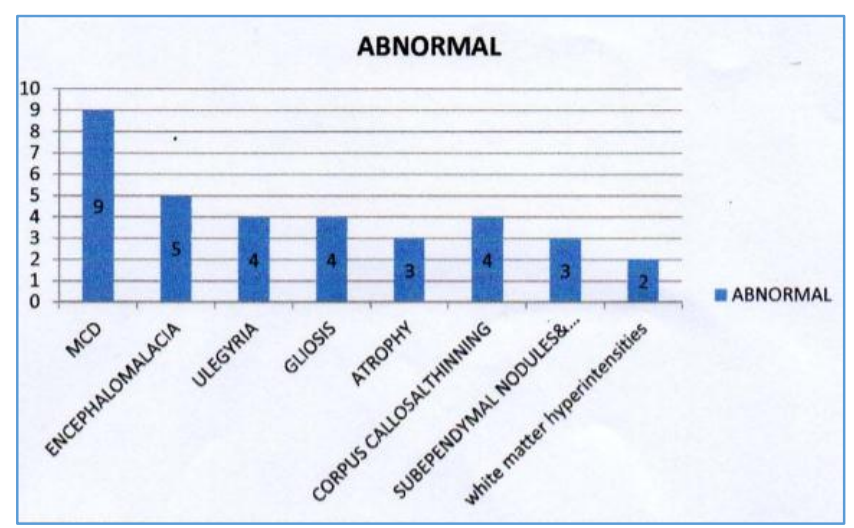

Figure 1

*Classification of MRI findings in abnormal group. †Malformations of Cortical Development (MCD) constituted $29.1 \%$ of the abnormal MRI findings.

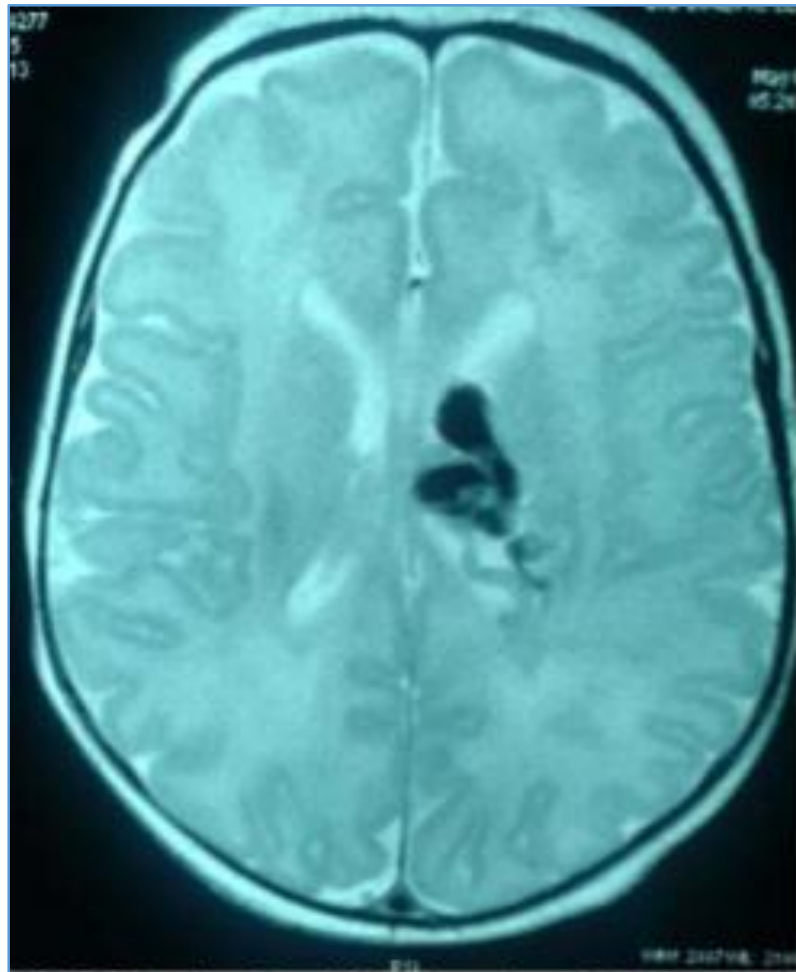

MRI Brain of a child showing Cavernoma

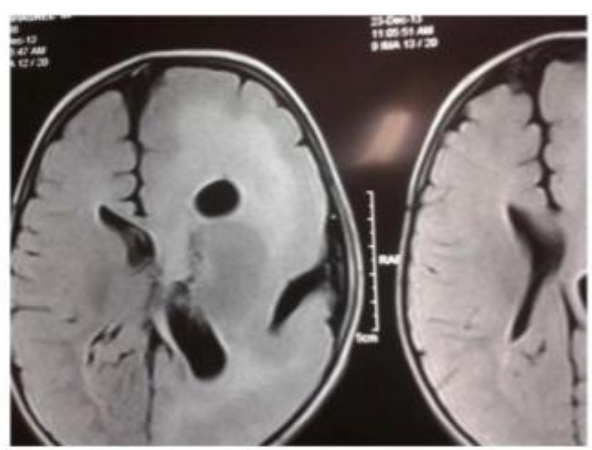

MRI Brain showing of a child with Epidermal Nevus Syndrome showing Left sided Hemimegalencephaly and Lissen cephaly

The age of onset of seizures for children with abnormal MRI was significantly lower than that of children with normal MRI. As the age of onset does not follow normality, the nonparametric test equivalent to test, i.e. Mann-Whitney $U$ test was also performed.

$53.8 \%$ of children with abnormal MRI and $51.2 \%$ of children with normal MRI had abnormal EEG. The difference was not found to be statistically significant. Out of abnormal EEG, generalised abnormalities (85.7\%) showed more association with MRI abnormalities than focal (14.3\%) cases. The difference was not statistically significant. 31 children with abnormal MRI had generalised seizures, whereas 5 had focal seizures and both types were present in 5 children. These observations were also not found to be statistically significant.

74.3\% children with history of status epilepticus had abnormal MRI findings, which was found to be statistically significant. Out of 13 children with abnormal head circumference, 9 had abnormal MRI. 15 children were on 2 or 
more AEDs and Intractable epilepsy; among them 10 had MRI abnormalities. Among 6 cases who had neonatal seizures, 5 babies had abnormal MRI. These associations were not statistically significant.

\section{DISCUSSION}

The study was conducted in Govt. Medical College, Thrissur, a tertiary care hospital in central Kerala. The aim of the study was to know the prevalence of MRI findings in children with epilepsy and to compare the findings in neurologically normal and abnormal groups. The study also aimed to identify the predictors or associations of abnormal MRI.

A total of 80 children in the age group of 2 to 14 years were enrolled in the study, out of which 40 children were with normal neurological examination and 40 were with abnormal neurological examination. Most of them were between 2 - 6 years of age (43\%).

The mean age was 7 years among 80 children, whereas in an Indian study by Chaurasia et al the predominant age group was between 13 and 18 years.(22) Gulati et al reports maximum number of cases between 6 and 12 years.(14) The difference could be due to the younger children predominantly attending the paediatric OPD, 2 - 6 years being included to a larger extent. Male children were the majority in a similar study by Ben Ameur et al.(23) While female children occupied the larger part in the study by Wongladarom et al.(24)

The mean age of onset of seizures was 4.15 years and median was 3 years, which was lesser compared to a study by Berg et al.(25) The mean age of onset was lower among children with abnormal examination findings. This difference might also be due to the predominance of the younger age group in the study population. Generalised Seizures were the predominant type of seizures seen with most of the studies from India.(5,21) Amirsalari et al in their cross-sectional study of 200 Iranian epileptic children reported predominance of focal seizures than generalised seizures.(26) Focal seizures constituted $79 \%$ of the seizure type in the study by Wongladarom et al.(24)

Epileptic syndromes were observed in only 10 patients (12.5\%), whereas Dura Trave et al reported high prevalence of the same.(27) Two cases, each of BECTS, CAE, Dravet Syndrome and four cases of Lennox-Gastaut Syndrome were present with normal MRI in three cases. One child with LGS had atrophic changes.

Intractable epilepsy was found in 10 children, which constituted (12.5\%). Berg et al(25) in a prospective study had shown comparable observation. Amirsalari et al reported positive family history in $56.5 \%$ and this series had only in $20 \%$.(26) $43 \%$ had a history of status epilepticus, either during presentation or later. Study by Berg et al in 613 American children showed status epilepticus in as little as $9.1 \%$. The difference could be due to the inclusion of both old and new cases.(25)

Berg et al reported abnormal neurological examination findings in $10.7 \%$ and similar in Amirsalari et al in 14.5\%, both studies included more patients with new-onset epilepsy. $50 \%$ of children in our series had abnormal findings, which was deliberately taken. A near comparable observation was seen in a study by Ben Ameur et al (67.3\%).(25) 52.5\% of the children showed EEG abnormalities, $50 \%$ in children with normal examination and 55\% in children with abnormal examination. Chaurasia et al reported abnormal EEG in $39.5 \% \cdot{ }^{(22)}$

Significant MRI abnormalities were detected in $48.75 \%$ of the study population. A few children had more than one abnormality, as described in a study by Dura Trave et al.(27) Higher percentage in a study by Gulati et al could be due to the inclusion of patients suspected to have an intracranial lesion.(14) Chaurasia et al reported abnormal MRI in 70.4\% cases; Ozateș et al described in $33.6 \%$ of children.(28) Wang et al studied 300 Chinese and reported similar results.(29)

Various studies have documented MRI abnormalities ranging between $16 \%-21 \%$ and the most common structural abnormality detected were congenital malformations neurocutaneous syndromes tumours and evidence of encephalopathy due to trauma, infection or asphyxia.(30)

Out of the 39 children with abnormal MRI the most common abnormality was malformations of cortical development, which constituted $23 \%$ followed by gliosis. Fourteen children had malformations of cortical development. Schizencephaly was the predominant one, followed by Focal cortical dysplasia and Nodular heterotopias. All children with schizencephaly had abnormal neurological examination, whereas children with focal cortical dysplasia had normal neurological examination. Wongladarom et al had similar observation with more number of children detected to have malformations of cortical development. Unlike other Indian studies by Chaurasia et al and Gulati et al where the predominant findings were CNS tuberculosis and Neurocysticercosis,(14,22) no such cases were there in the present study. This observation could be due to the geographical peculiarity, small sample size and the strict exclusion of acute symptomatic seizures. Sequelae of destructive injuries (hypoxia, ischaemia and hypoglycaemia) constituted 33\% of MRI abnormalities, which included Encephalomalacia, Gliosis and Ulegyria similar to a study of Ben Ameur et al.(23)

Neonatal hypoglycaemic brain injury (NHBI) was responsible for epilepsy in $7.5 \%$ among our series. Udani et al reports $23 \%$ of children with neonatal hypoglycaemic brain injury in the study conducted in children less than 3 years with symptomatic epilepsy.(31) All the children had lesions in the parieto-occipital region, comparable to the observation by Barkovich et al.(32)

Mesial temporal sclerosis constituted only $4.1 \%$ of the abnormal MRI findings. Thailand study by Wongladarom et al reports a higher percentage.(24) Three cases of Tuberous sclerosis with subependymal nodules and tubers, an epidermal nevus syndrome with hemimegalencephaly, a case of cavernoma and a hypothalamic hamartoma were present in this study. Chaurasia et al observed more number of phakomatoses and tumours.(22)

All of the benign idiopathic epileptic syndromes showed normal MRI except a case of BECTS, which showed Hippocampal sclerosis that is uncommon in literature.(33) The children with MTS and with FCD were referred for surgical management. MRI abnormalities showed a significant association with abnormal neurological examination like other studies.

MRI abnormalities showed a significant inverse relationship with the age of onset of seizures. Younger the age of onset, more children had abnormal MRI. This finding was comparable to Dura Trave et al, Ben Ameur et al and 
Berg et al.(25) Ben Ameur et al studied 140 Tunisian epileptic children and observed that the age of onset of seizures below 3 years was a predictive factor for abnormal MRI.(23)

Amirsalari et al reported significant relationship between abnormal EEG and abnormal MRI findings, whereas no association was observed with abnormal EEG, more than one type of seizure or focal seizures in this study. Abnormal MRI findings showed significant association with presence of status epilepticus in this study series (74.3\%). Study by Berg et al shows abnormalities in $27.5 \%$ only and this could be due to the difference in inclusion criteria.(25) Neonatal seizures, presence of more than one type of seizure or abnormal head circumference etc. did not show any association with abnormal MRI findings.

\section{Limitations}

The sample size was small. Age distribution of the two groups were different. There was no uniformity in the timing of MRI. Infants were not represented and adolescents were only a minority.

\section{CONCLUSION}

1. Magnetic Resonance Imaging abnormalities were more common among epileptic children with abnormal neurological examination.

2. The most common abnormality detected was Malformations of Cortical Development.

3. Children with lower age of onset, particularly those below 2 years showed more abnormalities.

4. Children with status epilepticus had more abnormalities on MRI.

5. MRI helps in management of children with underlying surgically amenable structural lesions.

\section{ACKNOWLEDGEMENTS}

1. *Dr. Rehna K. Rehman - Data collection, interpretation and analysis of the study.

2. *Dr. Sheela T.A - Study design, analysis of data, overall guidance and drafting the manuscript.

3. *Dr. Shaji Abhraham - Professor, Department of Neurology, Government Medical College, Thrissur (Helped in designing the study).

4. *Dr. Sudhiraj - Assistant Professor, Department of Social and Preventive Medicine, Government Medical College, Thrissur (Helped in statistical analysis).

\section{REFERENCES}

[1] Kliegman R. Nelson textbook of paediatrics. 19th edn. Elsevier/Saunders 2011; p. 2610.

[2] Swaiman KF, Ashwal S, Ferriero DM. Pediatric neurology: principles \& practice. Vol. 1. $4^{\text {th }}$ edn. Philadelphia, PA: Mosby 2006.

[3] Guidelines for diagnosis and management of childhood $\quad 2014$. http://medind.nic.in/ibv/t09/i8/ibvt09i8p681.pdf

[4] Fisher RS, Acevedo C, Arzimanoglou A, et al. ILAE official report: a practical clinical definition of epilepsy. Epilepsia 2014;55(4):475-82.

[5] Sridharan R, Murthy BN. Prevalence and pattern of epilepsy in India. Epilepsia 1999;40(5):631-6.
[6] Radhakrishnan K, Pandian JD, Santhoshkumar T, et al. Prevalence, knowledge, attitude, and practice of epilepsy in Kerala, South India. Epilepsia 2000;41(8):1027-35.

[7] Berg AT, Scheffer IE. New concepts in classification of the epilepsies: entering the 21st century. Epilepsia 2011;52(6):1058-62.

[8] Shorvon SD. The etiologic classification of epilepsy. Epilepsia 2011;52(6):1052-7.

[9] Birbeck GL. Revising and refining the epilepsy classification system: priorities from a developing world perspective. Epilepsia 2012;53(Suppl 2):18-21.

[10] Fisher RS, Cross JH, French JA, et al. Operational classification of seizure types by the International league against epilepsy: position paper of the ILAE commission for classification and terminology. Epilepsia 2017;58(4):522-30.

[11] Wright NB. Imaging in epilepsy: a paediatric perspective. Br J Radiol 2001;74(883):575-89.

[12] Deuschl, Gunther, Eisen, et al. Recommendations for the practice of clinical neurophysiology. Guidelines of the International Federation of Clinical Neurophysiology - NLM Catalog - NCBI 2014. http://www.ncbi.nlm.nih.gov/nlmcatalog/100891320

[13] American Clinical Neurophysiology Society. Guideline 1: minimum technical requirements for performing clinical electroencephalography. J Clin Neurophysiol 2006;23(2):86-91.

[14] Gulati P, Jena A, Tripathi RP, et al. Magnetic resonance imaging in childhood epilepsy. Indian Pediatr 1991;28(7):761-5.

[15] Kuzniecky RI. Neuroimaging in pediatric epilepsy. Epilepsia 1996;37(Suppl 1):S10-S21.

[16] Zupanc ML. Neuroimaging in the evaluation of children and adolescents with intractable epilepsy: II. Neuroimaging and paediatric epilepsy surgery. Pediatric Neurology 1997;17(2):111-21.

[17] Gaillard WD, Chiron C, Cross JH, et al. Guidelines for imaging infants and children with recent-onset epilepsy. Epilepsia 2009;50(9):2147-53.

[18] Barth PG. Disorders of neuronal migration. Can J Neurol Sci 1987;14(1):1-16.

[19] Palmini A, Holthausen H. Focal malformations of cortical development: a most relevant etiology of epilepsy in children. Handb Clin Neurol 2013;111:54965.

[20] Razek AAA, Kandell AY, Elsorogy LG, et al. Disorders of cortical formation: MR imaging features. American Journal of Neuroradiology 2008;30(1):4-11.

[21] Kuzniecky R, Murro A, King D, et al. Magnetic resonance imaging in childhood intractable partial epilepsies: pathologic correlations. Neurology 1993;43(4):681-7.

[22] Chaurasia R, Singh S, Mahur S, et al. Imaging in pediatric epilepsy: spectrum of abnormalities detected on MRI. Journal of Evolution of Medical and Dental Sciences 2013;2(19):3377-87.

[23] Ameur BS, Aloulou H, Sfaihi L, et al. Cerebral imaging in epileptic children: study of 140 cases. Tunis Med 2014;92(1):24-8. 
[24] Wongladarom S, Laothamatas J, Visudtibhan A, et al. Magnetic resonance imaging of the brain in epileptic pediatric patients: review of the experience in Ramathibodi hospital. J Med Assoc Thai 2004;87(9):1092-9.

[25] Berg AT, Testa FM, Levy SR, et al. Neuroimaging in children with newly diagnosed epilepsy: a communitybased study. Paediatrics 2000;106(3):527-32.

[26] Amirsalari S, Saburi A, Hadi R, et al. Magnetic resonance imaging findings in epileptic children and its relation to clinical and demographic findings. Acta Medica Iranica 2012;50(1):37-42.

[27] Durá-Travé T, Yoldi-Petri ME, Esparza-Estaún J, et al. Magnetic resonance imaging abnormalities in children withepilepsy. Eur J Neurol 2012;19(8):1053-9.

[28] Ozateş M, Acar M, Başak F. Cranial MRI findings in epileptic children. Tani Girisim Radyol 2003;9(4):42731.
[29] Wang PJ, Liu HM, Fan PC, et al. Magnetic resonance imaging in symptomatic/cryptogenic partial epilepsies of infants and children. Zhonghua Min Guo Xiao Er Ke Yi Xue Hui Za Zhi 1997;38(2):127-36.

[30] Berg AT, Shinnar S, Levy SR, et al. Early development of intractable epilepsy in children: a prospective study. Neurology 2001;56(11):1445-52.

[31] Udani V, Munot P, Ursekar $M$, et al. Neonatal hypoglycemic brain - injury a common cause of infantile onset remote symptomatic epilepsy. Indian Pediatr 2009;46(2):127-32.

[32] Barkovich AJ, Ali FA, Rowley HA, et al. Imaging patterns of neonatal hypoglycemia. AJNR Am J Neuroradiol 1998;19(3):523-8.

[33] Gelisse P, Corda D, Raybaud C, et al. Abnormal neuroimaging in patients with benign epilepsy with centrotemporal spikes. Epilepsia 2003;44(3):372-8. 